\title{
Neuroimaging in meningiomas: old tips and new tricks
}

\author{
Andrea Elefante ${ }^{1}$, Camilla Russo ${ }^{1}$, Martina Di Stasi ${ }^{1}$, Elena Vola ${ }^{1}$, Lorenzo Ugga ${ }^{1}$, Fabio Tortora ${ }^{1}$, Oreste De \\ Divitiis $^{2}$
}

\begin{abstract}
'Department of Advanced Biomedical Sciences, Division of Neuroradiology, University "Federico II", Naples 80131, Italy. ${ }^{2}$ Department of Neurosciences and Reproductive and Odontostomatological Sciences, Division of Neurosurgery, University "Federico II", Naples 80131, Italy.
\end{abstract}

\begin{abstract}
Correspondence to: Prof. Andrea Elefante, Department of Advanced Biomedical Sciences, Division of Neuroradiology, University "Federico II", Via S. Pansini 5, Napoli 80131, Italy. E-mail: aelefant@unina.it
\end{abstract}

How to cite this article: Elefante A, Russo C, Di Stasi M, Vola E, Ugga L, Tortora F, De Divitiis O. Neuroimaging in meningiomas: old tips and new tricks. Mini-invasive Surg 2021;5:7. http://dx.doi.org/10.20517/2574-1225.2020.102

Received: 5 Nov 2020 Accepted: 5 Nov 2020 Published: 3 Feb 2021

Academic Editor: Giulio Belli Copy Editor: Cai-Hong Wang Production Editor: Jing Yu

\begin{abstract}
Meningiomas are the most common neoplasm of the central nervous system. Usually benign and generally discovered incidentally at imaging, meningiomas can also be responsible for severe neurological symptoms and deficits, with potentially high morbidity and non-negligible mortality. Therefore, neuroimaging plays a crucial role in meningiomas diagnosis, therapeutic planning, and long-term surveillance, for early detection of both recurrence in treated patients and disease progression in untreated ones. Here, we review conventional findings in meningiomas' imaging, review the role for advanced diagnostic techniques, and offer an overview on possible future neuroimaging applications.
\end{abstract}

Keywords: Meningioma, magnetic resonance imaging, computed tomography, central nervous system

\section{INTRODUCTION}

Meningiomas account for about 36\% of all intra-cranial neoplasms, thus representing the most common primary tumor of the central nervous system $(\mathrm{CNS})^{[1]}$. They take origin from meningeal membranes covering brain, nerves, and spinal cord, arising from arachnoid mater formed by the cells within middle meningeal layer; therefore, this type of neoplasm, although more common in intra-cranial space, can be found all over the neuroaxis [Figure 1] $]^{[2,3]}$. More frequent in elderly (peak incidence in 6 th-7th

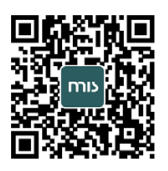



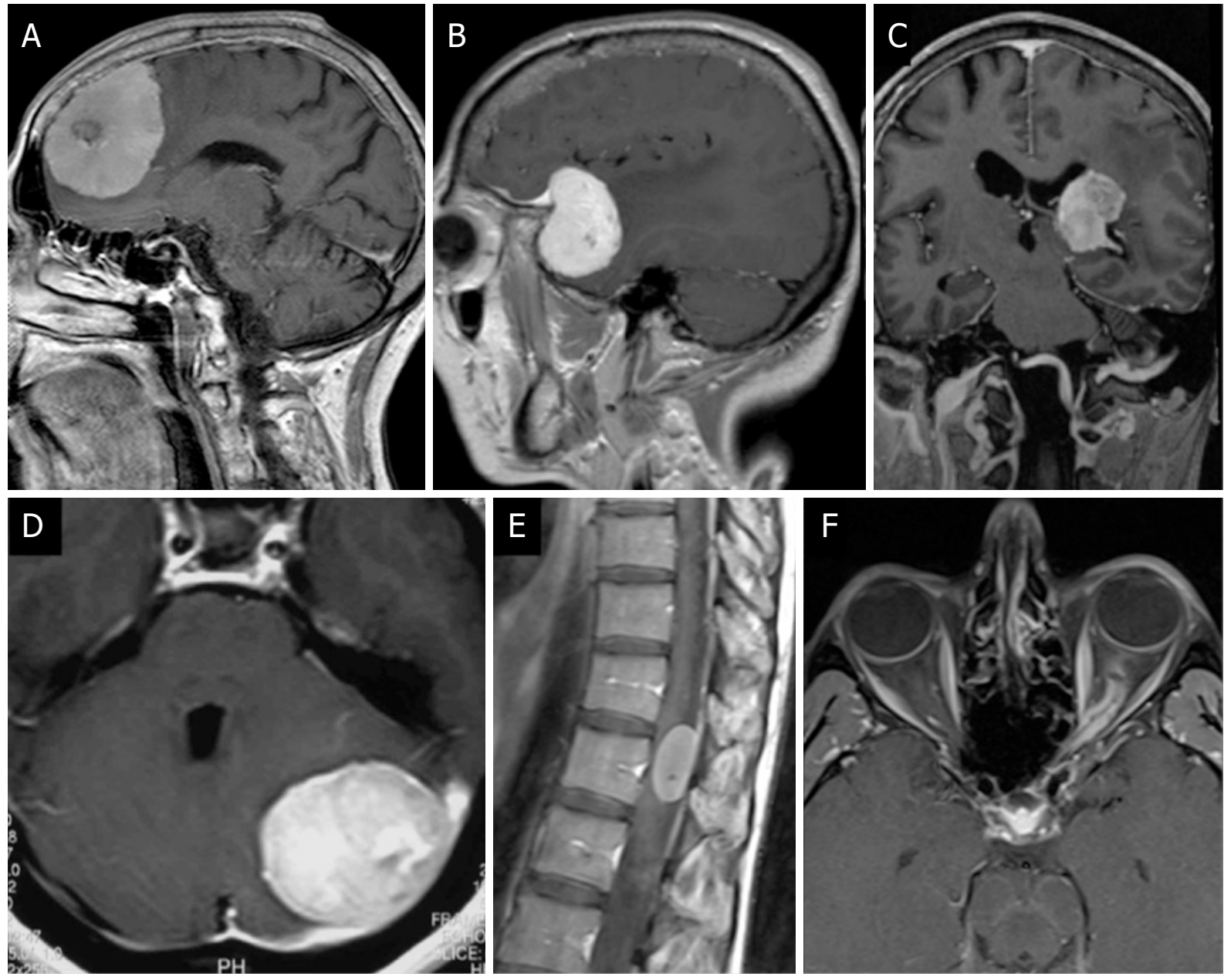

Figure 1. Post-contrast T1w images showing some of the possible different localizations of meningiomas along the neuroaxis: falx cerebri (A); sphenoid wing (B); intra-ventricular (C); tentorium (D); dorsal spine (E); and left optic nerve (F)

decades) and female patients (at least in part due to endogenous estrogen stimulation), their incidence is higher in the case of ionizing radiation exposure and in familiar predisposing syndromes such as Type 2 neurofibromatosis; in these latter cases, they are generally multiple, with more severe symptoms and common atypical locations (for example, intraventricular or at the skull base) ${ }^{[1,4]}$.

Meningiomas are usually benign and slow-growing extra-axial tumors with poor tendency to metastatic dissemination and local aggressiveness. Due to their relatively benign biologic behavior, meningiomas are frequently discovered incidentally during CNS imaging and for smaller ones a watchful waiting strategy can be envisaged. However, despite largely being considered a purely benign disease, meningiomas can also cause high morbidity and mortality due to focal neurological deficits, potentially difficult surgical resectability, and local aggressiveness; these features can cause extremely severe repercussions in terms of symptoms severity, functional limitations, and quality of life ${ }^{[1]}$. Due to this variability, the most recent 2016 World Health Organization (WHO) classification of CNS tumors proposed a revision of meningiomas classification, including the presence of necrosis, brain invasion, high cellularity, and elevated mitotic index with increase in small cells composition as diagnostic criteria for atypical meningiomas (Grade IIIII tumors ${ }^{[5]}$. While atypical high-grade meningiomas are associated to a worst prognosis, higher survival rates are reported for low-grade meningiomas; however, also in these cases neurological deficits and longterm disability are a common complication. 
With this knowledge, neurosurgical gross-total resection still represents the gold standard for patients' treatment, with radiotherapy used as adjuvant treatment in the case of non-radically removed lesions (whereas external-beam radiation was not demonstrated to be associated to better results compared to surgery followed by adjuvant radiation $)^{[6,7]}$. Surgical planning largely relies on MRI and CT scans examination, as the type of surgery performed can vary depending on tumor size and location. Different surgical techniques have been used for meningiomas. The most common approach is represented by craniotomy, in which brain exposure ensures tumor visualization on the brain surface, minimizing the risk of damaging adjacent structures. Another possible alternative is represented by neuroendoscopy-assisted microscopic resection techniques. Neuroendoscopic surgery is largely used for meningiomas within the ventricular chambers, whereas for ventral skull base meningiomas a possible option is represented by endoscopic endonasal surgery; however, while for olfactory groove or tuberculum sellae this latter approach has been widely validated, its use remains controversial in other skull base regions (such as cavernous sinus, petro-clival, or cranio-facial regions). Moreover, since meningiomas obtain vascular supply from extracranial and intracranial circulation, preoperative embolization can be used in selected cases as adjuvant therapy to reduce intraoperative bleeding and make surgery more effective; specific imaging techniques (such as perfusion and angiography) can provide information on meningioma's perfusion status, amenability to embolization based on blood supply, and eventual anatomical references that could help in the delivery of embolic materials. After partial resection, the disease-free survival rates range between about $60 \%$ at five years and $10 \%$ at 15 years, with ever-increasing tendency to recurrence over time. Nevertheless, also in the case of complete surgical removal, the overall rate of meningioma recurrence remains not negligible, as it is estimated to range $15 \%-25 \%$ at 20 years ${ }^{[8,9]}$.

With this background, it can be easily understood why neuroimaging plays a crucial role not only in meningioma first diagnosis, but also in therapeutic planning and long-term surveillance (for early detection of both recurrence in treated patients and disease progression in untreated ones). Here, we review the conventional findings in meningioma imaging, discuss the role of advanced diagnostic techniques, and offer an overview on possible future neuroimaging applications for lesions' characterization.

\section{CONVENTIONAL IMAGING}

\section{Intracranial meningiomas}

Intracranial meningiomas typically show characteristic neuroimaging features well detected on both CT and MRI studies, which allow the correct diagnosis with high diagnostic accuracy. MRI is the gold standard technique for meningiomas detection and evaluation because it provides soft tissue characterization, high contrast definition and possibility of multiplanar reconstructions ${ }^{[10]}$. Meningiomas appear as extraaxial dural-based masses, with the exception of en plaque meningioma, exhibiting sheet-like appearance due to its extensive dural extrinsecation ${ }^{[11]}$. On conventional MRI, they usually are hypo- to isointense on T1-weighted sequences, with variable signal on T2-weighted sequences due to the presence of necrotic cystic or calcific areas; most of them are avidly and homogeneously enhancing after paramagnetic agents administration [Figure 2] ${ }^{[12,13]}$. T2 w images also allow for crescent-shaped cerebral-spinal fluid (CSF) cleft between tumor and brain parenchyma identification, while post-contrast sequences allow for the detection of the characteristic dural tail, due to adjacent dural reactive changes ${ }^{[14]}$. Edema in surrounding brain tissue is evident in about half of cases, generally due to the presence of atypical features related to a more aggressive biological behavior rather than to overall dimension ${ }^{[15]}$; on diffusion-weighted images (DWI), brain edema is typically vasogenic, due to different mechanisms such as venous obstruction, pial vessel paralysis, and vessel barrier alteration ${ }^{[16-19]}$. DWI has also been used to depict higher-grade meningiomas with increased cellularity, which show reduced values on corresponding apparent diffusion coefficient (ADC) maps ${ }^{[20-22]}$; however, it should be noted that the correlation between DWI and tumor grade remains controversial, as no univocal statistical correlation between ADC values and tumoral behavior has been established yet ${ }^{[23]}$. Other imaging characteristic that have been proposed as indicative of a more aggressive 

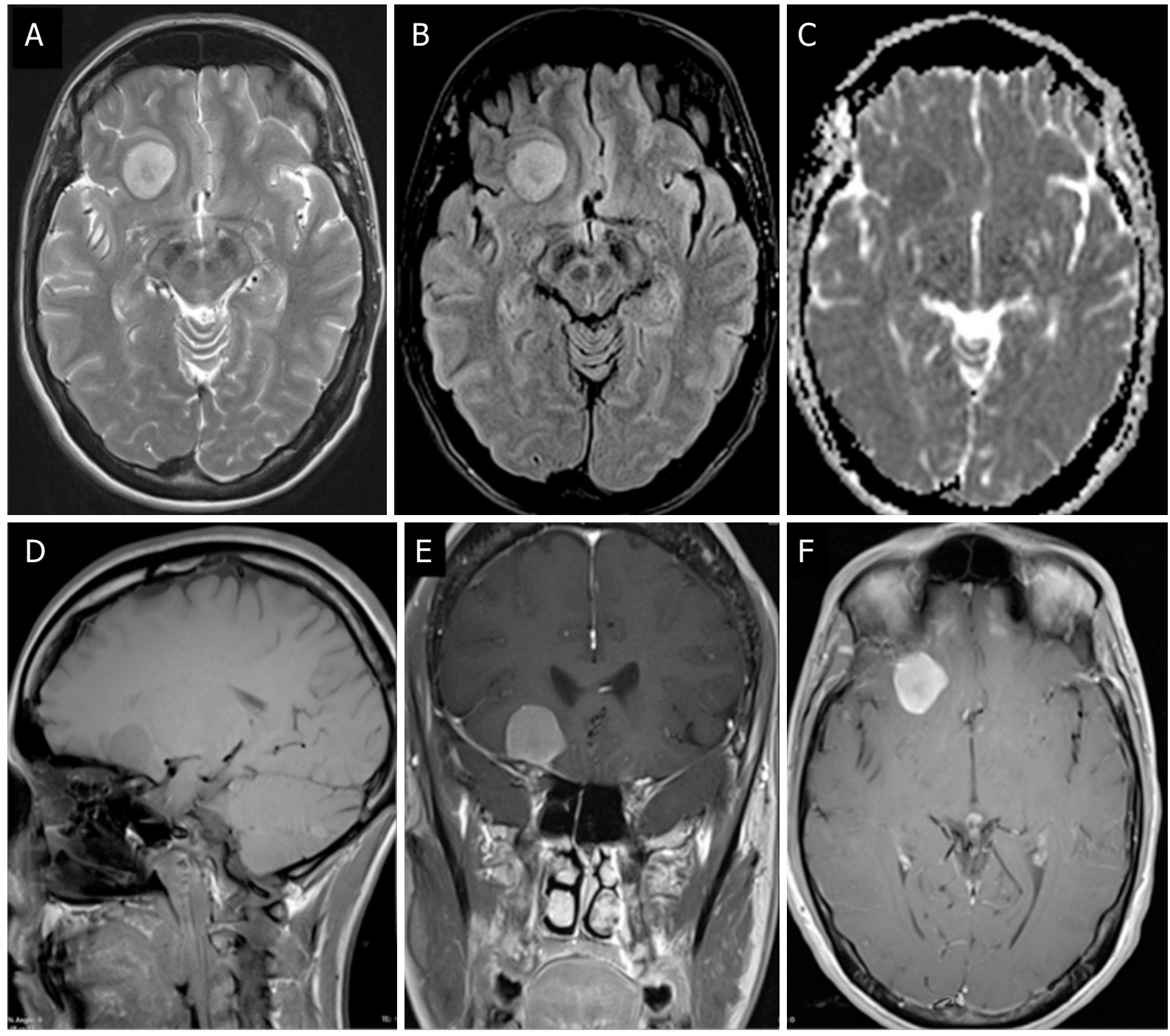

Figure 2. Right frontal low-grade meningioma on conventional MRI: hyperintense on T2W (A) and FLAIR (B) images, with no peripheral edema and a subtle crescent-shaped CSF cleft between tumor and adjacent brain tissue; no significant DWI restriction with moderateto-high value on ADC map compared to brain parenchyma (C); and iso-hypointense relative to cerebral grey matter on T1w (D), with homogeneous and intense post-contrast enhancement (E, F). CSF: cerebral-spinal fluid; DWI: diffusion-weighted images; ADC: apparent diffusion coefficient

behavior include irregular margins, undefined tumor-brain interface, intra-tumoral necrosis and cysts, and absence of calcifications on susceptibility-weighted sequences [Figure 3] ${ }^{[24]}$. Along with MRI, CT remains the gold standard for the depiction of tumor-inducted osseous changes such as remodeling with focal hyperostosis and bone thickening or bone invasion with associated osteoblastic reaction (more rarely osteolysis) in malignant cases ${ }^{[25]}$. Finally, meningiomas are highly vascularized tumors, being the blood supply provided by meningeal or vertebral-basilar branches; intra-tumoral dysplastic vessels can be better characterized in unenhanced and contrast-enhanced MR angiography. Conversely, MR venogram is usually performed to study venous sinuses invasion thrombosis or occlusion; while unenhanced phasecontrast MR venogram (and also black-blood MR imaging) has been demonstrated as a reliable method in assessing sinus invasion, it should always be considered that higher sensitivity in detecting collateral anastomoses and draining veins around the lesion is obtained with contrast-enhanced MR venography. This information is important both for surgical planning and for sinus preservation in the case of radiotherapy/ radiosurgery $^{[17-19]}$. 

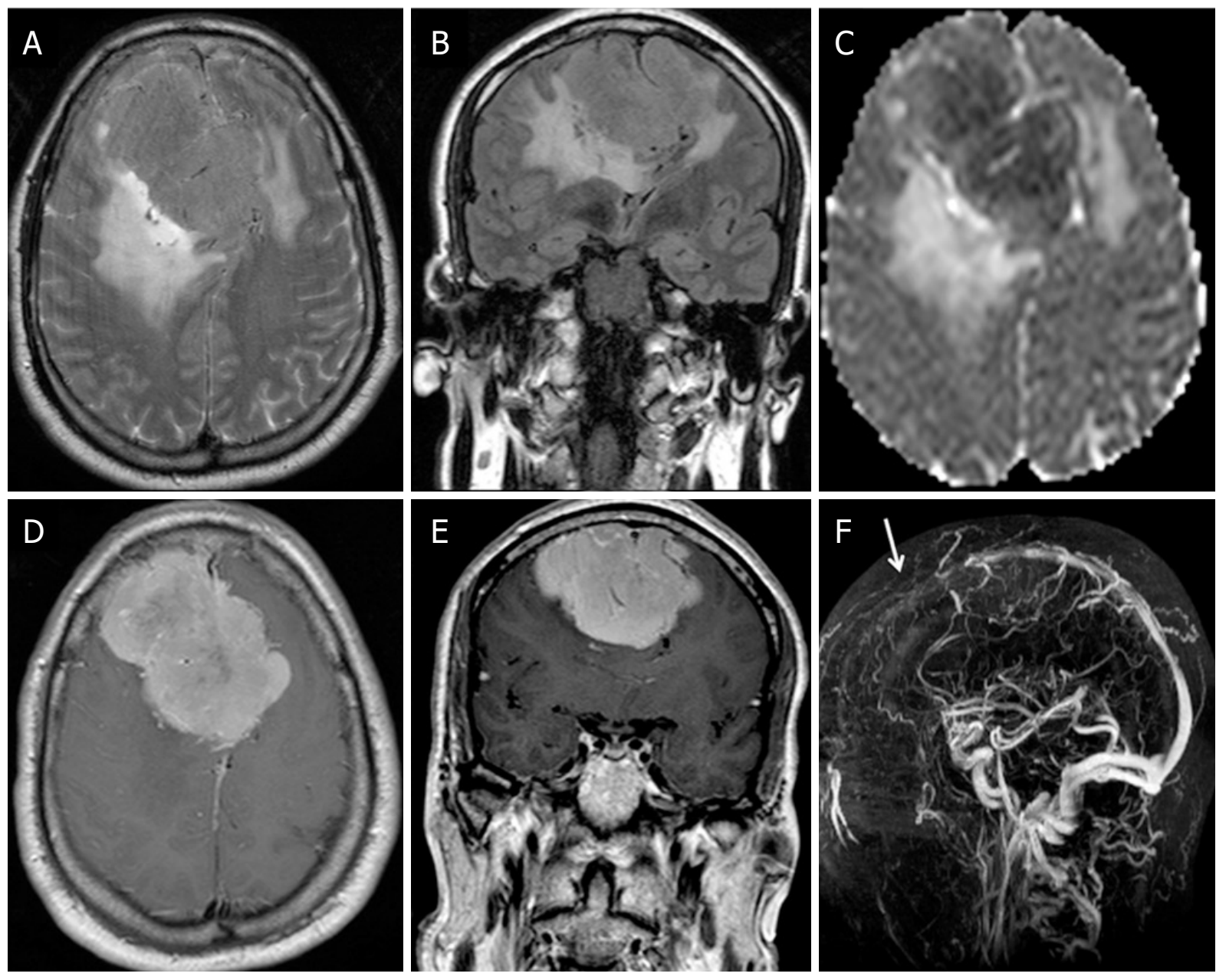

Figure 3. Falx cerebri high-grade meningioma on conventional MRI: inhomogeneous on T2w due to calcifications and necrosis ( $A$ ), with large peripheral edema halo and no clear distinction from normal brain tissue on FLAIR (B); lower values compared to normal brain parenchyma on $A D C$ maps (C); intense and inhomogeneous post-contrast enhancement after i.v. gadolinium administration (D, E); and invasion of the anterior segment of sagittal sinus on 3D PCA venogram (F). ADC: apparent diffusion coefficient

\section{Spinal meningiomas}

Spinal meningiomas are extra-spinal intra-dural well-defined masses, with only few cases arising from epidural compartment with both extra- and intra-dural extension (the latter are usually more aggressive, with higher risk of recurrence) ${ }^{[26,27]}$. MRI of the spine represents the modality of choice for both diagnosis and follow-up; characteristics are similar to intra-cranial meningiomas, with a slightly lower signal on T2w compared to the spinal cord ${ }^{[28]}$. Calcifications are less common than in intra-cranial compartment and more often reported in epidural lesions. Yeo et al. ${ }^{[2]}$ classified spinal meningiomas in four main subgroups, based on neuroimaging features: intradural homogeneous neoplasm avidly enhancing, with or without dural tail (Type A); round tumors with hypointense area on T2w images (Type B); en plaque meningiomas with a collar-like growth along spinal cord (Type C); and other meningiomas with atypical features (Type $D)^{[29,30]}$. Epidural meningioma, a rare entity classified as Type D, is often misdiagnosed due to its peculiar location; typical enlargement of neuroforamina determined by epidural growth pattern can be used as differential diagnostic feature ${ }^{[31]}$.

\section{ADVANCED TECHNIQUES: POSSIBLE APPLICATIONS}

Conventional MRI generally responds adequately to diagnostic purposes, however differential diagnosis between extra-axial dural-based masses (or between different meningioma subtypes) can be very 
challenging. Tissue characterization, identification of important features for surgical planning, and prognostic biomarkers individuation can be enhanced by the use of advanced imaging techniques.

\section{Spectroscopy}

Spectroscopy is an MRI technique used to assess metabolite concentration in a region of interest. Therefore, it can be used for differential diagnosis both to differentiate between intra- and extra-axial masses and to exclude the hypothesis of dural metastases in the case of extra-axial dural-based mass in oncologic patients. Meningiomas show elevated choline and decreased $\mathrm{N}$-acetylaspartate as well as decreased creatinine, a metabolic profile common to other neoplastic processes and therefore quite unspecific; conversely, increased alanine has demonstrated to be specific for meningioma but can be difficult to identify ${ }^{[32,33]}$. An elevated metabolite peak at 3.8 parts per million has been described in meningiomas, allowing to differentiate them from high-grade gliomas and intracranial metastasis. MR spectroscopy has been demonstrated to not be able to differentiate atypical meningiomas from typical ones ${ }^{[34,35]}$. Lactate peak is considered suggestive of aggressiveness, but it can also be found in benign meningiomas. Nevertheless, lactate and macromolecular peaks have demonstrated significant differences in meningothelial, fibrous, and oncocytic subtypes, showing the potential to characterize various lesion components ${ }^{[36]}$.

\section{Perfusion imaging}

MR perfusion is a technique used to assess blood flow in tissues and includes the dynamic susceptibility contrast (DSC) technique and the dynamic contrast enhancement (DCE) technique, both requiring the administration of intravenous gadolinium, and arterial spin labeling. Meningiomas are highly vascular lesions, deriving their blood supply from meningeal arteries and consequently demonstrating very high perfusion. The complete lack of the blood-brain barrier determines increased contrast leakage and permeability, represented by a typical time-intensity curve: rapid drop during the first pass of contrast and slow return to a level lower than brain parenchyma ${ }^{[37]}$. MR perfusion can be useful in differential diagnosis, in particular to differentiate meningiomas from dural-based metastases and from high-grade gliomas invading the dura mater. Indeed, MR perfusion may differentiate between meningioma and dural metastases from various origins (breast, colon, and prostate) but not from hypervascular metastases, such as those from melanoma, renal carcinoma, or Merkel cell carcinoma (increased cerebral blood volume ${ }^{[38]}$. The assessment of the time-intensity curve can distinguish a primary glial neoplastic process from intracranial metastases/meningiomas: in the former, the curve shows more than $50 \%$ return to baseline, while, in the latter, the curve shows less than $50 \%$ return to baseline due to breakdown in blood-brain barrier and dural-based blood supply. Meningioma vascularity appears to be significantly related to cerebral blood flow $(\mathrm{CBF})$ values ${ }^{[39-41]}$ and lately a significant correlation between CBV and expression of vascular endothelial growth factor has also been demonstrated, suggesting the possibility to use perfusion MR to predict refractoriness to conventional treatment and possible responsiveness to anti-angiogenic therapies. Correlation between relative $\mathrm{CBV}(\mathrm{rCBV})$ and Ki67 proliferative index has also been demonstrated in meningiomas but several studies have shown contrasting results about a possible correlation between tumoral perfusion parameters and meningioma grade, probably because of increased vascular permeability of meningiomas, due to lack of blood-brain barrier ${ }^{[2,42,43]}$. On the contrary, peritumoral rCBV has shown a potential diagnostic role: although peritumoral rCBV usually shows decreased values in meningiomas, possibly due to peritumoral vasogenic edema ${ }^{[44]}$, its values are higher in the case of anaplastic meningiomas (WHO Grade III) compared with the other types ${ }^{[45]}$. Similarly, decreased peritumoral CBF can be measured with CT perfusion, potentially representing ischemic tissue salvageable after meningioma resection ${ }^{[46]}$. Arterial spine labeling has the advantage of assessing perfusion without the confounding permeability influence, potentially allowing to differentiate WHO Grade I from WHO Grades II and III intracranial meningiomas ${ }^{[47]}$. Vascular permeability represents another measurable parameter, assessed directly via DCE technique and contributing to meningioma grading: atypical meningiomas have shown higher values of Ktrans compared with benign meningiomas ${ }^{[48]}$. MR perfusion can be helpful also in distinguishing some 
meningioma subtypes. In particular, angiomatous meningioma has demonstrated higher tumor rCBV compared with meningothelial, fibrous, or anaplastic subtypes ${ }^{[45]}$.

\section{Diffusion tensor imaging}

Given the possibility to assess magnitude and directionality of water diffusion, diffusion tensor imaging (DTI) has been applied to differentiate meningioma grades. Although in most studies high-grade meningiomas have demonstrated low ADC values when compared with low-grade ones, controversial results have been obtained especially for the other DTI parameters ${ }^{[49-51]}$. DTI has shown promising potential in terms of preoperative consistency prediction. Besides some contrasting findings, most studies have shown higher fractional anisotropy (FA) values in hard meningiomas compared to soft ones ${ }^{[52-54]}$. Signal intensity on FA and mean diffusivity maps have also been found to be predictive of meningioma consistency ${ }^{[52,53,55]}$. Tractography, derived from DTI data, may give additional information for treatment planning of skull base meningiomas, but it is usually not necessary: resolving the course of cranial nerves with CSF sensitive sequences is technically easier and less sensitive to $\operatorname{artifacts}^{[12]}$.

\section{MR elastography}

MR elastography (MRe) is a promising emerging technique that may have the potential to define tumor consistency and its relationship with adjacent structures. It provides a measurement of tissue stiffness, determined by the assessment of share wave movement through that given tissue. Recent studies have demonstrated a significant correlation between the MRe measurements and intraoperative qualitative assessment of tumor consistency ${ }^{[33]}$. Furthermore, differing stiffness on both sides of a tissue boundary allow defining the measurement of freedom of the adjacent tissue planes, thus evaluating the marginal invasiveness ${ }^{[56]}$.

\section{Molecular imaging}

The most used molecular imaging technique in oncological field is $2-{ }^{[18 \mathrm{~F}]}$-fluoro-2-deoxy-D-glucose (18F-FDG)-PET, which uses a glucose analog to identify metabolically active cells, but it does not have a primary role in intracranial tumors diagnosis due to high physiological FDG uptake in cerebral cortex and FDG accumulation in inflammatory processes. The ability of FDG-PET to differentiate meningioma grades has shown contrasting results. Although some studies have demonstrated its ability to differentiate benign meningioma from atypical/malignant ones and to distinguish recurrent/growing meningiomas from static ones, there is a lack of correlation between FDG uptake and WHO grading, MIB-1 labeling index, and tumor doubling time $e^{[12]}$. On the other hand, a high meningioma-to-background contrast can be obtained using radiolabeled somatostatin receptors II (SSTR II) ligands due to the increased expression of SSTR II in meningiomas compared to the very low expression in bone and brain tissue $e^{[57,58]}$. PET with gallium-68labeled SSTR-ligands, such as 68Ga-DOTATOC (DOTA-(Tyr3)-octreotid) and 68Ga DOTATATE (DOTADPhe1-Tyr3-octreotate), has demonstrated a higher sensitivity in detecting meningiomas when compared to contrast enhanced MRI ${ }^{[59]}$. SSTR-PET is also useful for differential diagnosis, for example when studying optic sheath meningioma ${ }^{[60]}$. This technique also allows a detailed meningioma extent delineation, necessary for treatment planning but challenging in the case of complex localization (skull base, orbit, falx cerebri, sagittal, and cavernous sinuses), trans-osseous growth, or in pre-treated meningiomas, when MR contrast results are limited ${ }^{[12,61]}$. Integration of SSTR-PET imaging increases the precision of resection and target radiation. Furthermore, SSTR-PET can differentiate viable tumor and scar tissue using a semi-qua ntitative data analysis, since semi-quantitative uptake values (SUV) correlate significantly with SSTR II expression assessed by immunostaining. Patient treatment stratification can take advantage of SSTR-PET since SUV measurements have also demonstrated a correlation with tumor growth rate in WHO Grades I and II meningiomas (not in Grade III). Furthermore, SSTR-PET has been demonstrated to be more specific for detecting residual meningioma and may be considered in the case of equivocal MRI findings ${ }^{[62-64]}$. Recently, the RANO-PET taskforce has proposed an evidence-based recommendation for the use of 
molecular imaging in meningiomas, even if the utility of SSTR II imaging needs more validation to be confirmed ${ }^{[65]}$.

\section{FUTURE DIRECTIONS}

Radiomics is an emerging field of research that extracts many features from medical images. There are two categories of features, which can be extracted from the region of interest after the lesion segmentation, semantic and agnostic ones. In detail, semantic features are commonly used in the radiology lexicon to describe a lesion (e.g., shape, location, etc.), but in the radiomics field they are quantified through computer assistance. On the other hand, diagnostic features describe lesion heterogeneity using quantitative descriptors. They include first-, second-, or higher-order statistics. First-order statistical outputs consist of the grey level histogram analysis of the lesion's voxels. Second-order statistics are those obtained from texture analysis. They describe relationships between voxels considering their contrast values. Finally, higher-order statistics are obtained imposing filters to extract definite image patterns, such as fractal analyses, wavelets, or Laplacian transforms of Gaussian bandpass filters ${ }^{[6]}$. Radiomics can be coupled with artificial intelligence, which employs algorithms to allow computers to learn directly from the data and make predictions on unseen datasets, because of its better capability of managing this volume of data compared to traditional statistics ${ }^{[6]}$. In the study of meningiomas, radiomics and artificial intelligence have shown promise in preoperative evaluation, recurrence and outcome prediction, and radiation treatment planning. Preoperative prediction of the meningioma grade is important because it influences the treatment strategy. Park et al ${ }^{[68]}$ obtained an accuracy of $89.7 \%$ for the prediction of meningioma grades using MR conventional and diffusion tensor imaging with a radiomics and machine learning approach; furthermore, various texture parameters differed significantly between fibroblastic and non-fibroblastic benign meningiomas. Volumetric assessment of meningiomas is also highly relevant for therapy planning and monitoring. Using a multiparametric deep-learning model on routine MRI data, Laukamp et al. ${ }^{[69]}$ investigated its performance in automated detection and segmentation of meningiomas in comparison to manual segmentations, obtaining a strong correlation despite diverse scanner data. Moreover, prognostic models based on clinical, radiologic, and radiomic feature have been investigated to preoperatively identify meningiomas at risk for poor outcomes. In this setting, preoperative radiologic and radiomic features such as apparent diffusion coefficient and sphericity have proved effective in predicting local failure and overall survival in these patients ${ }^{[70]}$. MR radiomics has also been implemented to predict early progression or recurrence, which characterize a subset of skull base meningiomas, achieving good results (accuracy $90 \%)^{[71]}$. Finally, radiomics has proved useful in the definition of radiotherapy target volume, which represents a critical step in treatment planning, in order to improve the texture-based differentiation of tumor from edema and to differentiate vasogenic from tumor cell infiltration edema ${ }^{[72]}$.

\section{CONCLUSION}

Although generally easily identified on the basis of some pictorial neuroimaging features, meningiomas can raise some concerns in terms of tissue characterization and treatment selection. In particular, surgery largely relies on MRI and CT scans examination, as the type of therapeutic approach can vary depending on tumor size and location. Modern imaging tools are helpful in identifying more aggressive histological behavior, defining vessel and brain involvement, and evaluating the need for adjuvant therapies; at the same time, emerging post-processing techniques can enhance tumor biology tracking and response to therapy prediction. All these imaging-derived data coupled together may allow for optimal therapeutic planning and tailored longitudinal follow-up, based on both patient and tumor fingerprinting.

\section{DECLARATIONS}

\section{Authors' contributions}

Made substantial contributions according to ICMJE criteria: Elefante A, Russo C, Di Stasi M, Vola E, Ugga L, Tortora F, De Divitiis O Conception and design: Elefante A 
Writing: Russo C, Di Stasi M, Vola E, Ugga L

Supervision: Tortora F, De Divitiis O

\section{Availability of data and materials}

Not applicable.

\section{Financial support and sponsorship}

None.

\section{Conflicts of interest}

All authors declared that there are no conflicts of interest.

\section{Ethical approval and consent to participate}

Not applicable.

\section{Consent for publication}

Not applicable.

\section{Copyright}

(c) The Author(s) 2021.

\section{REFERENCES}

1. Goutagny S, Bah AB, Henin D, et al. Long-term follow-up of 287 meningiomas in neurofibromatosis type 2 patients: clinical, radiological, and molecular features. Neuro Oncol 2012;14:1090-6.

2. Buerki RA, Horbinski CM, Kruser T, Horowitz PM, James CD, Lukas RV. An overview of meningiomas. Future Oncol 2018;14:2161-77.

3. Mariniello G, Briganti F, De Caro ML, Maiuri F. Cervical extradural "en-plaque" meningioma. J Neurol Surg A Cent Eur Neurosurg 2012;73:330-3.

4. Hashiba T, Hashimoto N, Izumoto S, et al. Serial volumetric assessment of the natural history and growth pattern of incidentally discovered meningiomas. J Neurosurg 2009;110:675-84

5. Louis DN, Perry A, Reifenberger G, et al. The 2016 World Health Organization Classification of Tumors of the Central Nervous System: a summary. Acta Neuropathol 2016;131:803-20.

6. Alghamdi M, Li H, Olivotto I, et al. Atypical meningioma: referral patterns, treatment and adherence to guidelines. Can J Neurol Sci 2017;44:283-7.

7. van Alkemade H, de Leau M, Dieleman EM, et al. Impaired survival and long-term neurological problems in benign meningioma. Neuro Oncol 2012;14:658-66.

8. Saraf S, McCarthy BJ, Villano JL. Update on meningiomas. Oncologist 2011;16:1604-13.

9. Jääskeläinen J. Seemingly complete removal of histologically benign intracranial meningioma: late recurrence rate and factors predicting recurrence in 657 patients. A multivariate analysis. Surgical Neurology 1986;26:461-9.

10. Islam $\mathrm{OH}$, Grayson, Coombs B, et al. Imaging in brain meningioma. 2014. Available from: http://emedicine. medscape.com/ article/341624-overview.2015. [Last accessed on 9 Nov 2020]

11. Watts J, Box G, Galvin A, Brotchie P, Trost N, Sutherland T. Magnetic resonance imaging of meningiomas: a pictorial review. Insights Imaging 2014;5:113-22.

12. Huang RY, Bi WL, Griffith B, et al; International Consortium on Meningiomas. Imaging and diagnostic advances for intracranial meningiomas. Neuro Oncol 2019;21:i44-61.

13. Ly KI, Wen PY, Huang RY. Imaging of Central Nervous System Tumors Based on the 2016 World Health Organization Classification. Neurol Clin 2020;38:95-113.

14. Buetow MP, Buetow PC, Smirniotopoulos JG. Typical, atypical, and misleading features in meningioma. Radiographics 1991;11:1087-106.

15. Sade B, Lee JH. High incidence of optic canal involvement in clinoidal meningiomas: rationale for aggressive skull base approach. Acta Neurochir (Wien) 2008;150:1127-32; discussion 1132.

16. Tamiya T, Ono Y, Matsumoto K, Ohmoto T. Peritumoral brain edema in intracranial meningiomas: effects of radiological and histological factors. Neurosurgery 2001;49:1046-51; discussion 1051-2.

17. Todua FI, Chedia SV, Nuralidze KI. Computed tomography and magnetic resonance angiography of brain meningiomas. Georgian Med News 2013;21-7.

18. Raza SM, Gallia GL, Brem H, Weingart JD, Long DM, Olivi A. Perioperative and long-term outcomes from the management of parasagittal meningiomas invading the superior sagittal sinus. Neurosurgery 2010;67:885-93; discussion 893. 
19. Mariniello G, Napoli M, Russo C, et al. MRI features of spinal solitary fibrous tumors. A report of two cases and literature review. Neuroradiol J 2012;25:610-6.

20. Hakyemez B, Yildirim N, Erdoğan C, Kocaeli H, Korfali E, Parlak M. Meningiomas with conventional MRI findings resembling intraaxial tumors: can perfusion-weighted MRI be helpful in differentiation? Neuroradiology 2006;48:695-702.

21. Watanabe Y, Yamasaki F, Kajiwara Y, et al. Preoperative histological grading of meningiomas using apparent diffusion coefficient at 3T MRI. Eur J Radiol 2013;82:658-63.

22. Tang Y, Dundamadappa SK, Thangasamy S, et al. Correlation of apparent diffusion coefficient with Ki-67 proliferation index in grading meningioma. AJR Am J Roentgenol 2014;202:1303-8.

23. Sanverdi SE, Ozgen B, Oguz KK, et al. Is diffusion-weighted imaging useful in grading and differentiating histopathological subtypes of meningiomas? Eur J Radiol 2012;81:2389-95.

24. Lee EJ, Kim JH, Park ES, et al. A novel weighted scoring system for estimating the risk of rapid growth in untreated intracranial meningiomas. J Neurosurg 2017;127:971-80.

25. O'Leary S, Adams WM, Parrish RW, Mukonoweshuro W. Atypical imaging appearances of intracranial meningiomas. Clin Radiol 2007;62:10-7.

26. Mariniello G, Malacario F, Dones F, et al. Sudden post-traumatic sciatica caused by a thoracic spinal meningioma. Neuroradiol $J$ 2016;29:390-2.

27. Sung CW, Hsieh KL, Kuo YJ. A primary meningioma of the lumbar spine with neck metastasis. J Spinal Cord Med 2019;1-4.

28. De Verdelhan O, Haegelen C, Carsin-nicol B, et al. MR imaging features of spinal schwannomas and meningiomas. J Neuroradiol 2005;32:42-9.

29. Yeo Y, Park C, Lee JW, et al. Magnetic resonance imaging spectrum of spinal meningioma. Clin Imaging 2019;55:100-6.

30. Zhang LH, Yuan HS. Imaging appearances and pathologic characteristics of spinal epidural meningioma. AJNR Am J Neuroradiol 2018;39:199-204.

31. Liu WC, Choi G, Lee SH, et al. Radiological findings of spinal schwannomas and meningiomas: focus on discrimination of two disease entities. Eur Radiol 2009;19:2707-15.

32. Tamrazi B, Shiroishi MS, Liu CS. Advanced imaging of intracranial meningiomas. Neurosurg Clin N Am 2016;27:137-43.

33. Shiroishi MS, Cen SY, Tamrazi B, et al. Predicting meningioma consistency on preoperative neuroimaging studies. Neurosurg Clin N Am 2016;27:145-54.

34. Demir MK, Iplikcioglu AC, Dincer A, Arslan M, Sav A. Single voxel proton MR spectroscopy findings of typical and atypical intracranial meningiomas. Eur J Radiol 2006;60:48-55.

35. Gajjar K, Heppenstall LD, Pang W, et al. Diagnostic segregation of human brain tumours using Fourier-transform infrared and/or Raman spectroscopy coupled with discriminant analysis. Anal Methods 2012;5:89-102.

36. Zakhari N, Torres C, Castillo M, Nguyen TB. Uncommon cranial meningioma: key imaging features on conventional and advanced imaging. Clin Neuroradiol 2017;27:135-44.

37. Newton AT, Pruthi S, Stokes AM, Skinner JT, Quarles CC. Improving perfusion measurement in DSC-MR imaging with multiecho information for arterial input function determination. AJNR Am J Neuroradiol 2016;37:1237-43.

38. Kremer S, Grand S, Rémy C, et al. Contribution of dynamic contrast MR imaging to the differentiation between dural metastasis and meningioma. Neuroradiology 2004;46:642-8.

39. Kimura $\mathrm{H}$, Takeuchi H, Koshimoto $\mathrm{Y}$, et al. Perfusion imaging of meningioma by using continuous arterial spin-labeling: comparison with dynamic susceptibility-weighted contrast-enhanced MR images and histopathologic features. AJNR Am J Neuroradiol 2006;27:85-93.

40. Hakyemez B, Erdogan C, Bolca N, Yildirim N, Gokalp G, Parlak M. Evaluation of different cerebral mass lesions by perfusion-weighted MR imaging. J Magn Reson Imaging 2006;24:817-24.

41. Cha S, Knopp EA, Johnson G, Wetzel SG, Litt AW, Zagzag D. Intracranial mass lesions: dynamic contrast-enhanced susceptibilityweighted echo-planar perfusion MR imaging. Radiology 2002;223:11-29.

42. Ginat DT, Mangla R, Yeaney G, Schaefer PW, Wang H. Correlation between dynamic contrast-enhanced perfusion MRI relative cerebral blood volume and vascular endothelial growth factor expression in meningiomas. Acad Radiol 2012;19:986-90.

43. Ginat DT, Mangla R, Yeaney G, Wang HZ. Correlation of diffusion and perfusion MRI with Ki-67 in high-grade meningiomas. AJR Am J Roentgenol 2010;195:1391-5.

44. Bitzer M, Klose U, Geist-Barth B, et al. Alterations in diffusion and perfusion in the pathogenesis of peritumoral brain edema in meningiomas. Eur Radiol 2002;12:2062-76.

45. Zhang Q, Jia GJ, Zhang GB, et al. A logistic regression model for detecting the presence of malignant progression in atypical meningiomas. World Neurosurg 2019;126:e392-401.

46. Sergides I, Hussain Z, Naik S, Good C, Miles K, Critchley G. Utilization of dynamic CT perfusion in the study of intracranial meningiomas and their surrounding tissue. Neurol Res 2009;31:84-9.

47. Qiao XJ, Kim HG, Wang DJJ, et al. Application of arterial spin labeling perfusion MRI to differentiate benign from malignant intracranial meningiomas. Eur J Radiol 2017;97:31-6.

48. Yang S, Law M, Zagzag D, et al. Dynamic contrast-enhanced perfusion MR imaging measurements of endothelial permeability: differentiation between atypical and typical meningiomas. AJNR Am J Neuroradiol 2003;24:1554-9

49. Nagar VA, Ye JR, Ng WH, et al. Diffusion-weighted MR imaging: diagnosing atypical or malignant meningiomas and detecting tumor dedifferentiation. AJNR Am J Neuroradiol 2008;29:1147-52.

50. Santelli L, Ramondo G, Della Puppa A, et al. Diffusion-weighted imaging does not predict histological grading in meningiomas. Acta 
Neurochir (Wien) 2010;152:1315-9; discussion 1319.

51. Wang S, Kim S, Zhang Y, et al. Determination of grade and subtype of meningiomas by using histogram analysis of diffusion-tensor imaging metrics. Radiology 2012;262:584-92.

52. Tropine A, Dellani PD, Glaser M, et al. Differentiation of fibroblastic meningiomas from other benign subtypes using diffusion tensor imaging. J Magn Reson Imaging 2007;25:703-8.

53. Kashimura $\mathrm{H}$, Inoue $\mathrm{T}$, Ogasawara $\mathrm{K}$, et al. Prediction of meningioma consistency using fractional anisotropy value measured by magnetic resonance imaging. J Neurosurg 2007;107:784-7.

54. Ortega-Porcayo LA, Ballesteros-Zebadúa P, Marrufo-Meléndez OR, et al. Prediction of mechanical properties and subjective consistency of meningiomas using T1-T2 assessment versus fractional anisotropy. World Neurosurg 2015;84:1691-8.

55. Romani R, Tang WJ, Mao Y, et al. Diffusion tensor magnetic resonance imaging for predicting the consistency of intracranial meningiomas. Acta Neurochir (Wien) 2014;156:1837-45.

56. Yin Z, Hughes JD, Trzasko JD, et al. Slip interface imaging based on MR-elastography preoperatively predicts meningioma-brain adhesion. J Magn Reson Imaging 2017;46:1007-16.

57. Reubi JC. Clinical relevance of somatostatin receptor imaging. Eur J Endocrinol 1994;131:575-6.

58. Reimold M, la Fougère C. Molecular imaging in neurological diseases. Radiologe 2016;56:580-7.

59. Afshar-Oromieh A, Giesel FL, Linhart HG, et al. Detection of cranial meningiomas: comparison of ${ }^{68}$ Ga-DOTATOC PET/CT and contrast-enhanced MRI. Eur J Nucl Med Mol Imaging 2012;39:1409-15.

60. Klingenstein A, Haug AR, Miller C, Hintschich C. Ga-68-DOTA-TATE PET/CT for discrimination of tumors of the optic pathway. Orbit 2015;34:16-22.

61. Nowosielski M, Galldiks N, Iglseder S, et al. Diagnostic challenges in meningioma. Neuro Oncol 2017;19:1588-98.

62. Sommerauer M, Burkhardt JK, Frontzek K, et al. 68Gallium-DOTATATE PET in meningioma: a reliable predictor of tumor growth rate? Neuro Oncol 2016;18:1021-7.

63. Slotty PJ, Behrendt FF, Langen KJ, Cornelius JF. (68)Ga-DOTATATE-positron emission tomography imaging in spinal meningioma. $J$ Craniovertebr Junction Spine 2014;5:44-6.

64. Rachinger W, Stoecklein VM, Terpolilli NA, et al. Increased 68Ga-DOTATATE uptake in PET imaging discriminates meningioma and tumor-free tissue. J Nucl Med 2015;56:347-53.

65. Galldiks N, Albert NL, Sommerauer M, et al. PET imaging in patients with meningioma-report of the RANO/PET Group. Neuro Oncol 2017;19:1576-87.

66. Gillies RJ, Kinahan PE, Hricak H. Radiomics: images are more than pictures, they are data. Radiology 2016;278:563-77.

67. Koçak B, Durmaz EŞ, Ateş E, Kılıçkesmez Ö. Radiomics with artificial intelligence: a practical guide for beginners. Diagn Interv Radiol 2019;25:485-95

68. Park YW, Oh J, You SC, et al. Radiomics and machine learning may accurately predict the grade and histological subtype in meningiomas using conventional and diffusion tensor imaging. Eur Radiol 2019;29:4068-76.

69. Laukamp KR, Thiele F, Shakirin G, et al. Fully automated detection and segmentation of meningiomas using deep learning on routine multiparametric MRI. Eur Radiol 2019;29:124-32.

70. Morin $\mathrm{O}$, Chen WC, Nassiri F, et al. Integrated models incorporating radiologic and radiomic features predict meningioma grade, local failure, and overall survival. Neurooncol Adv 2019;1:vdz011.

71. Zhang Y, Chen JH, Chen TY, et al. Radiomics approach for prediction of recurrence in skull base meningiomas. Neuroradiology 2019;61:1355-64.

72. Florez E, Nichols T, E Parker E, T Lirette S, Howard CM, Fatemi A. Multiparametric magnetic resonance imaging in the assessment of primary brain tumors through radiomic features: a metric for guided radiation treatment planning. Cureus 2018;10:e3426. 